\title{
THE IMPLEMENTATION OF THE HUMAN COMPUTER INTERACTION SYSTEM BASED ON BIOELECTRICITY
}

\author{
Sharda Shalikrao Kakde \\ Department Of Computer Science and Engineering) \\ Walchand College Of Engineering (An Autonomous Institute) \\ Sangli, India \\ Dr. Bashirahamad F Momin \\ Department Of Computer Science and Engineering) \\ Walchand College Of Engineering (An Autonomous Institute) \\ Sangli, India
}

\begin{abstract}
The electroencephalogram (EEG) evaluates brain waves, whereas the electrooculogram (EOG) evaluates blinking eye signals. To use bioelectric signals in bio-metric and clinical applications, preprocessing of the signal needs to be done. Signals are used to transmit data in nearly every sector of life including technology, manufacturing, and electronics, etc. Nowadays HCI is based on bioelectric signal has got loads of demand. Almost every sector bioelectrical signals are used especially in the medical sector and researches. In this paper, two bioelectric signals are used that is EEG, EOG. Two HCI (Human-Computer Interaction) systems were designed which is based on two kinds of bioelectric signals is EOG and EEG. The signal is transmitted by wireless mode only because in a wired HCI System user is not comfortable. Here HCI system contains three sections first is signal acquisition and signal transmission module second is EOG and EEG handling module and the last one is function implementing modular. This paper deals with the concentration level and meditation level of a person which is very useful in sports and other researches.
\end{abstract}

Index Terms-Brain-Computer-Interface, Human-ComputerInteraction, Electromyography, Electrooculogram, Wireless, Wired.

\section{INTRODUCTION}

$\mathrm{HCI}$ is an interaction between computers and humans. It is a study that is based on the design of computer science and the interaction between humans and computers. While initially concerned with computers. EOG, ECG, EMG, and EEG all measure biopotentials that are bioelectricity. EEG, ECG, EMG nothing but the electrical activity of a person. It is broadly applied to many sectors for example medical treatments, medical science, robotics, and entertainment.
Bioelectricity is the electrical flows and electrical possibilities created by or happening inside living cells, tissues, and organisms. Bioelectricity is the electrical wonders of life processes. All cells are electric.

EOG: Electrooculogram (EOG) is a method of measuring the condition of the cornea before and after the human eye. The resulting mark is called an electrooculogram. Mainly used in eye surgery and recording human eye movements.

EEG: A graphical record (EEG) is a test that involves inserting little metal disks (electrodes) on the scalp to live electrical activity within the brain. Brain cells communicate through electrical impulses and are continuously active even throughout sleep.

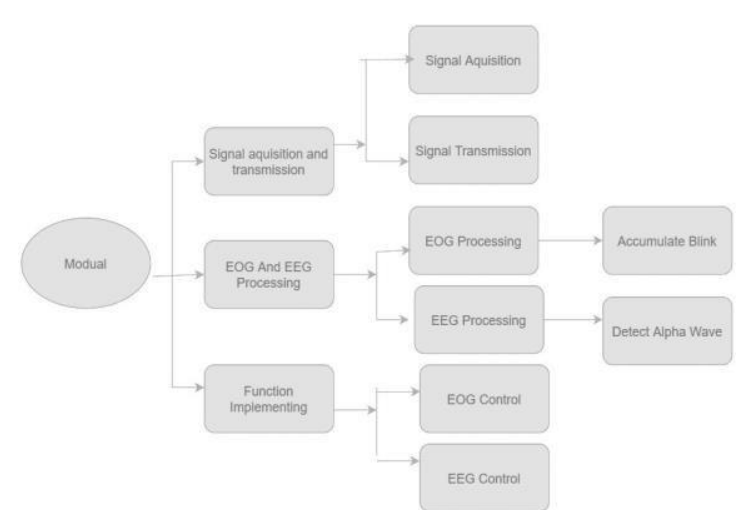

Fig. 1. System Modular diagram

This activity is shown as a wavy line in the EEG recording. The EEG is one of the foremost necessary diagnostic tests for encephalopathy. The EEG is used to diagnosis different brain diseases The EEG can observe changes in brain activity and facilitate determine brain diseases especially epilepsy (a neurological disorder) and other diseases. The EEG can also be used to diagnose or treat the following 


\section{International Journal of Engineering Applied Sciences and Technology, 2021 \\ Vol. 6, Issue 2, ISSN No. 2455-2143, Pages 105-110 \\ Published Online June 2021 in IJEAST (http://www.ijeast.com)}

diseases: brain damage caused by brain tumors and traumatic brain injury.

\section{LITERATURE REVIEW}

According to Kenji $\mathrm{Y}$, Junichi $\mathrm{H}$, Michio $\mathrm{M}$ in [1] for unable to speak, a communication support interface controlled by eye movement and autonomous blinking was developed. horizontal and vertical electrooculograms were measured with two electrodes placed above and on the side of the main eye. were from earlobe electrodes and amplified with $\mathrm{AC}$ coupling to reduce unnecessary drift. The direction cursor movement and selection operation are performed by a logical combination of two sensing channel signals set based on respective thresholds. As shown by the results of Runs using the projected on- screen keyboard, the processing speed increased from to 12.1 letters/minute. The accuracy of is 90.4 .

S. Venkataramanan, Pranay Prabhat, Shubhodeep Roy Choudhury, Harshal B. Nemade, J.S. Sahambi proposed that [2], the Electrooculogram (EOG) signal is obtained by signal acquisition framework and conspicuous antiques and obstruction circular segment sUPI. There are more than 4 million disabled individuals in India. For a useful switch that can be used by handicapped people as a warning. Patients who are admitted to medical clinics frequently have issues with working gadgets physically. This might be expected to stability brought about by mishaps and removal, or body sicknesses, for example, loss of motion and polio. A set of buttons are placed around the head or neck and controlled by the customer according to a limited development curve. Available to him. Due to the easy work of the staff and the moderately calm atmosphere of the clinic, this undoubtedly improves the unshakable quality, so today's clinics in the commercial market have an alarm framework based on customer voicecommands. Individual patients rely on various language features, such as pitch, pitch, volume, and repeatability.

Yoshitsugu Yasui proposed in [3], the implications of evaluating the usability of NeuroSky MindBuilder-EM (MB). Before that, due to its size and complexity, most braincomputer interfaces (BCIs) had not been fully used for clinical and research purposes. MB is a single anode earplug based on electroencephalogram (EEG) measurement, which receives weak electrical signals generated by neurodevelopment. The electrical signal passing through the terminal is evaluated to select the level. Life assessment work. The evaluation suggests that MB provides an accurate measurement of thinking because there is a positive correlation between the level of conscious thinking and selfreport G. The results also show that there are some specific adaptability and problems in its sports. Proposed future assessments, including the importance of standardized procurement and calculation methods, to offset the usual volatility in customer thinking when used as a source of information.
Genaro Rebolledo-Mendez, Ian Dunwell and Sara de Freitas have presented in [4], they integrated consumer EEG equipment with an EEG cathode cap to create an easy, small, and remote electroencephalogram (EEG) framework suitable for field chronicles. While members stepped outdoors on campus grounds, they were given audible crazy information. A single preliminary P300 order combined with direct discriminant analysis revealed strong characterization exactnesses for both indoor and outdoor environments (77 percent).

According to Debener S, Minow F, Emkes R, Gandras K, de Vos M., in [5] They combined consumer EEG kits with EEG electrode caps. When the participants walked outside the campus, they made an acoustically strange discovery. They concluded that the existing equipment can provide high-quality single-shot EEG test data suitable for mobile brains and computer interfaces. The study included healthy volunteers who had no previous or current neurological or mental illnesses.

Adarsh Rajesh, and Megha Mantur prsented in[6],They developed a working prototype based only on a small camera and a microprocessor that displays more information. They will also show them the significant improvements in the performance of traditional imaging algorithms, which will enable these patients to gain more independence in their daily lives and significantly improve their quality of life at an affordable price.

Sitthichai Iampetch,Yunyong Punsawad [7] in this study, The prediction of mental exhaustion using the electroencephalogram (EEG) has been extensively studied. The EEG definitely changes when you feel tired. Challenge, however, is that accurate fatigue prediction results consist of real-time selection of the EEG interval of interest. This article proposes a novel method to efficiently select the EEG signal during the period of fatigue as a marker by the electrooculogram (EOG).

Matthew K. Mukerjee [8], They will introduce the design, implementation and evaluation of the NeuroPhone system, which uses neural signals to control the iPhone's mobile phone application through cheap, off-the-shelf wireless electroen- cephalogram (EEG) hearing aids. They showed a mind-driven address book dialing application that works similarly to the user interface of the P300speller brain-computer: the phone displays a series of photos of contacts in the address book. If the displayed photos determine that the P300's brain potential matches the user's Who. Want to call. The EEG signal from the headset is wirelessly transmitted to the iPhone.

\section{METHODOLOGY}

The method includes three stages: Collection and transmission of modular signals, EOG and modular EEG processing, Modularization functions.

\section{A. Collection and transmission of modular signals}


This is the first step, there are two assemblies are the right side and left side assembly. As we can see in Fig 2, in the left side assembly, In this project I use Neurosky Mindwave Mobile 2 Brainwave Starter Kit, there is one sensor that should touch on the skin above our left eyebrow with a little pressure. Sensors measure the voltage fluctuation that place contains all thoughts. After that attach ear clip on-ear, which measures heartbeat rate. After that, it generates the waves. There are two meters at the bottom in the hardware kit which generate attention and meditation of a person. In right-side assembly, I use a hardware kit for EOG (the kit includes small parts of hardware that is a synthesizer, An Arduino, HC-05 Bluetooth Module, capacitors, resistors, electrodes, wires), I took a piece of thermal and place the hardware on that in a Y shape. Attach data cable to the one side of aurdino and second side to the power bank. In that hardware there are 4 wires, attach 2 wires above the right eyebrow and two below the right eye. Then open the kit. After completing all the steps, we will obtain the original signal data we need, and the next operation will be to store and process the signal. Here we use EEG Dataset from Kaggle for ideal data values.

1) Filtering is designed with a 3-pole $4 \mathrm{~Hz}$ Butterworth filter and a 3-pole $30 \mathrm{~Hz}$ Butterworth filter.

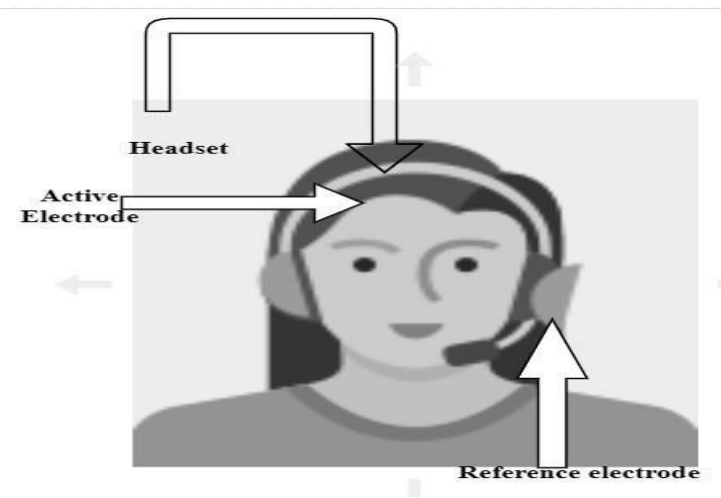

Fig. 2. The Experiment Environment

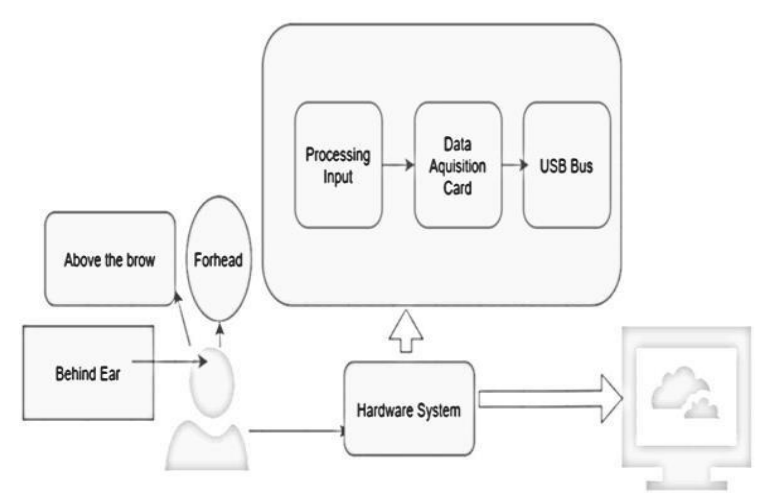

Fig. 3. Collection And Transmission Of Modular Signals

2) The complex number result of the output function of the FFT system is stored in a complex number matrix, and the real part of each element records the amplitude and The index indicates the frequency parameter.

3) The peak frequency of the brain wave is accumulated. Get the fundamental frequency through filtering and FFT. The next step is to determine whether the fundamental frequency band is in the middle $(8 \mathrm{~Hz} 12 \mathrm{~Hz})$ for 3 seconds.

\section{B. EOG and EEG Processing Modual}

EOG processing unit: The signal we get from our process is not clean, because the EOG frequency is mainly concentrated in the bandwidth below $10 \mathrm{~Hz}$, the waveform of the signal be- comes smoother and there is less frequency interference. The next step is to recognize the blinking action and accumulate the continuum. Blink based on EOG waveform features seven main types of EOG features can be used to identify Blink movement can be identified by seven types of EOG features.

EEG processing unit: Due to the difference in frequency and amplitude, psychological waves are usually divided into 5 bands, such as delta wave with frequency $(0.1 \mathrm{~Hz} 3$ $\mathrm{Hz})$, wave with frequency $(4 \mathrm{~Hz} 7 \mathrm{~Hz})$ and alpha wave. Band frequency $(8 \mathrm{~Hz} 12 \mathrm{H})$, Beta. Waves with frequency $(13 \mathrm{~Hz} 30 \mathrm{~Hz})$ and gamma waves with frequency $(31 \mathrm{~Hz}$ $100 \mathrm{~Hz}$ ). Knowing that the human brain tends to relax naturally, particularly once the

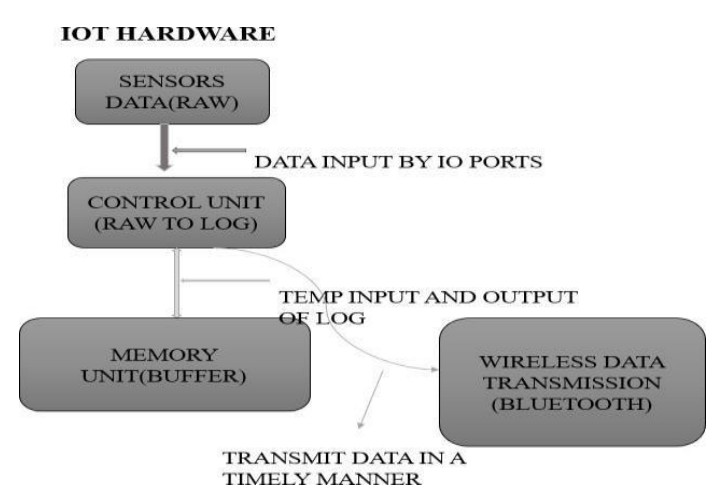

Fig. 4. Working Of IOT HARDWARE

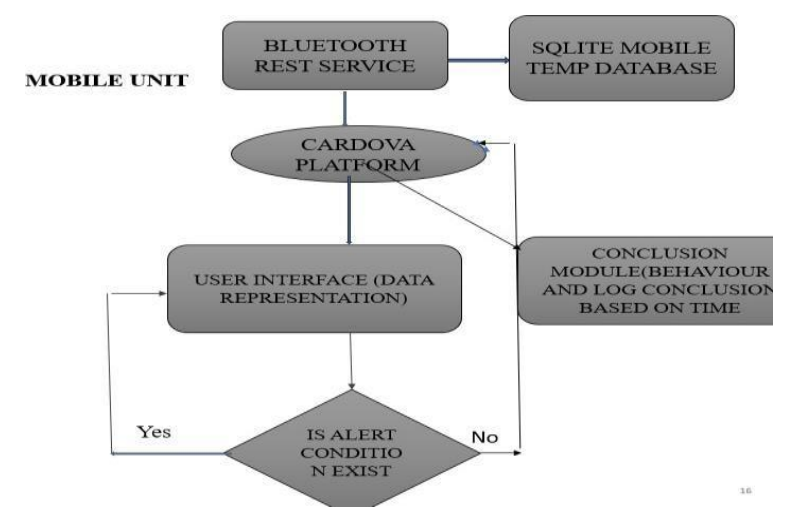

Fig. 5. Working Of Mobile Unit

eyes are closed, the alpha rhythm becomes stronger, 


\section{International Journal of Engineering Applied Sciences and Technology, 2021 \\ Vol. 6, Issue 2, ISSN No. 2455-2143, Pages 105-110 \\ Published Online June 2021 in IJEAST (http://www.ijeast.com)}

therefore it may be known and recorded. As a part of the graph, there are 3 stages of designing and execution: C. Function Implementing Module during this framework, the module contains 2 units: the EOG unit and also the EEG work unit. The interaction object is a Bluetooth. Fig. 4 and fig. 5 represents the working of IOT Hardware and working of mobile unit.

There are 3 phases of development: Phase 1, In this we studied existing technology to read EOG and EEG and develop a combination of hardware module which can transmit data to an android phone via Bluetooth. Phase 2, In this we will build and mobile interface for capturing data signals transmitted by Phase 1 hardware modules. The next is designing internal algorithms to analyze and equate results based on inputs along with user experience. All the time of development one Machine Learning platform will analyze data for further processing. Phase 3 , is the phase of utility where we bring out real-life action based on the conclusion of phase2.

Hardware used in this project is Neurosky Brainwave Mobile Kit, an Arduino, an Android Smartphone with Bluetooth, HC-05 Bluetooth Module.

\section{PROBLEM STATEMENT}

Implementation of human computer interaction system based on bio-electrical signal (EEG, EOG).

Due to the growing demand for highly secure personal identification in many applications in our highly networked society, human identity verification depend on bio electrical signals is a fast developing research field. They are used not only for medical diagnostic purposes, but also for human body verification because they have unique characteristics among humans. This article examines new research on bioelectrical signals such as the electrocardiogram (ECG), electroencephalogram (EEG), and electrooculogram (EOG) (EOG). The utilisation of bioelectric signals in human body authentication creates a novel user authentication approach that incorporates all of the key features of traditional authentication.

\section{RESULTS AND DISCUSSION}

In this study, we are developing an android application with a Bluetooth interface. To test the applicability to real brainwave measurements, two types of tests were selected. The first attempt was a continuous measurement in normal life, including night sleep, and the second was a dedicated driving application. In the continuous measurement, the subject user is a male, age is 31 . The measurement is taken during a typical day. In fig 6 , we can see a normal brain waves look like as alpha, beta gamma, theta, delta.

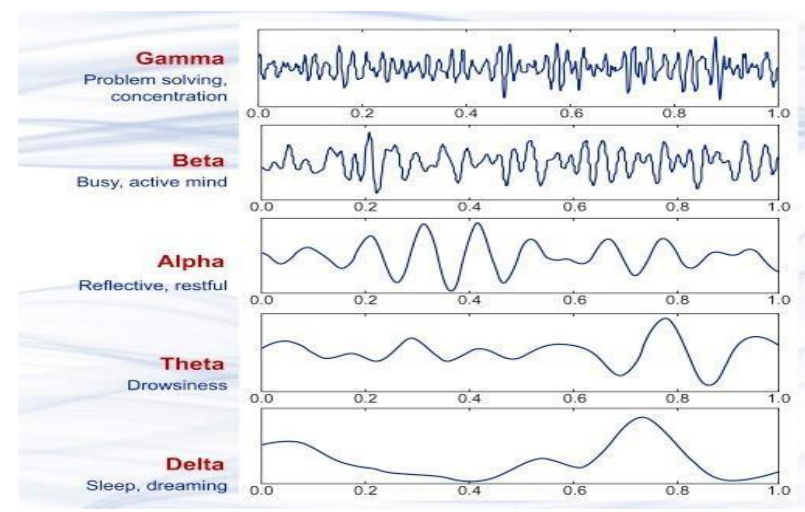

Fig. 6. Brain waves

Frequency band Frequency Brain states Gamma (Concentration), Beta (Anxiety dominant, active, external attention, relaxed),

Alpha (Very relaxed, passive attention) Theta (Deeply relaxed, inward focused) Delta (Sleepy) The names of the electrode sites are abbreviated alphabetically to designate the area of the brain from which each electrode records:

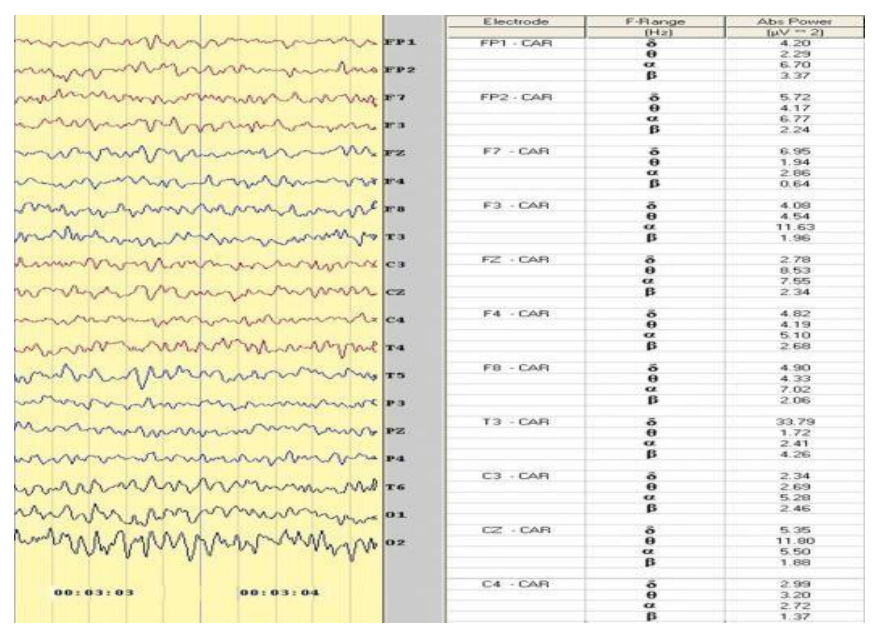

Fig. 7. The Corresponding Results In The System

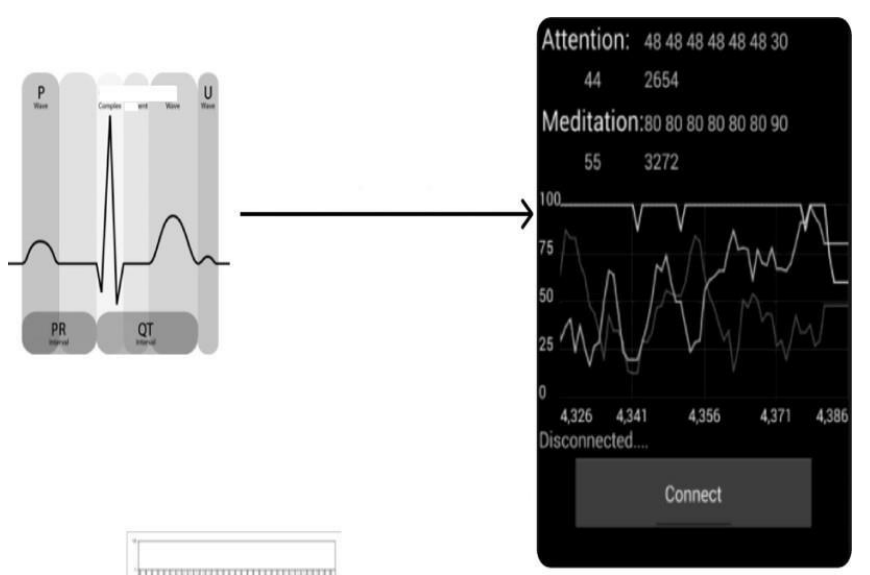

Fig. 8. Reading Progress 1 
As we see in Fig 9, in the Reading Progress, the first wavelike image is related to the output from the sensor which is $\mathrm{P}$ wave, $\mathrm{R}$ wave combines called PR interval. PR Interval- $\mathrm{R}$ is normally a straight line and $\mathrm{P}$ is Pulse. After that, we find QRS, T Wave. We called QT intervals and These are electronic pulses that are the combination of brain and heart bit traveling. After that convert it to waves that are With the help of Delta wave, Theta wave, Alpha wave, Mu wave, SMR wave, Beta wave, Gamma wave. In the rightside diagram 2654 is the summary and 44 is the average value. In attention, there is a high frequency and in meditation, there is a low frequency. We get these scores with the help of the equation as follows. here $\mathrm{fl}=$ delta wave, $f 2=$ theta, and alpha is a straight line, hence we cant consider it in our equation. After that, we get a cos value because we want a cos value to make a graph.

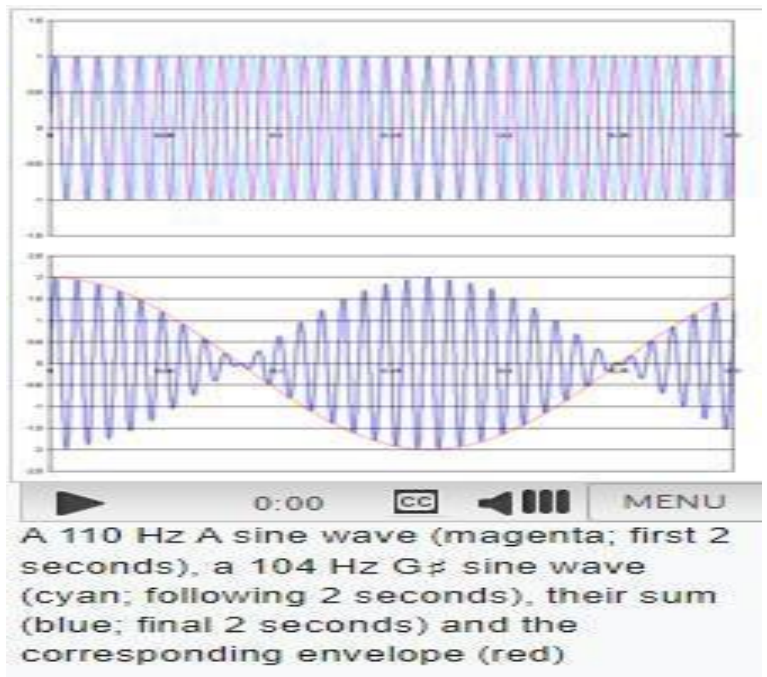

Fig. 9. Reading Progress 2

Fig 10, represents the snapshot of android application. Ideal dataset doesn't change which is in red colour graph and blue colour dataset change as per user connection. If score is less then the meditation level is high and if score is high then concentration level is high.

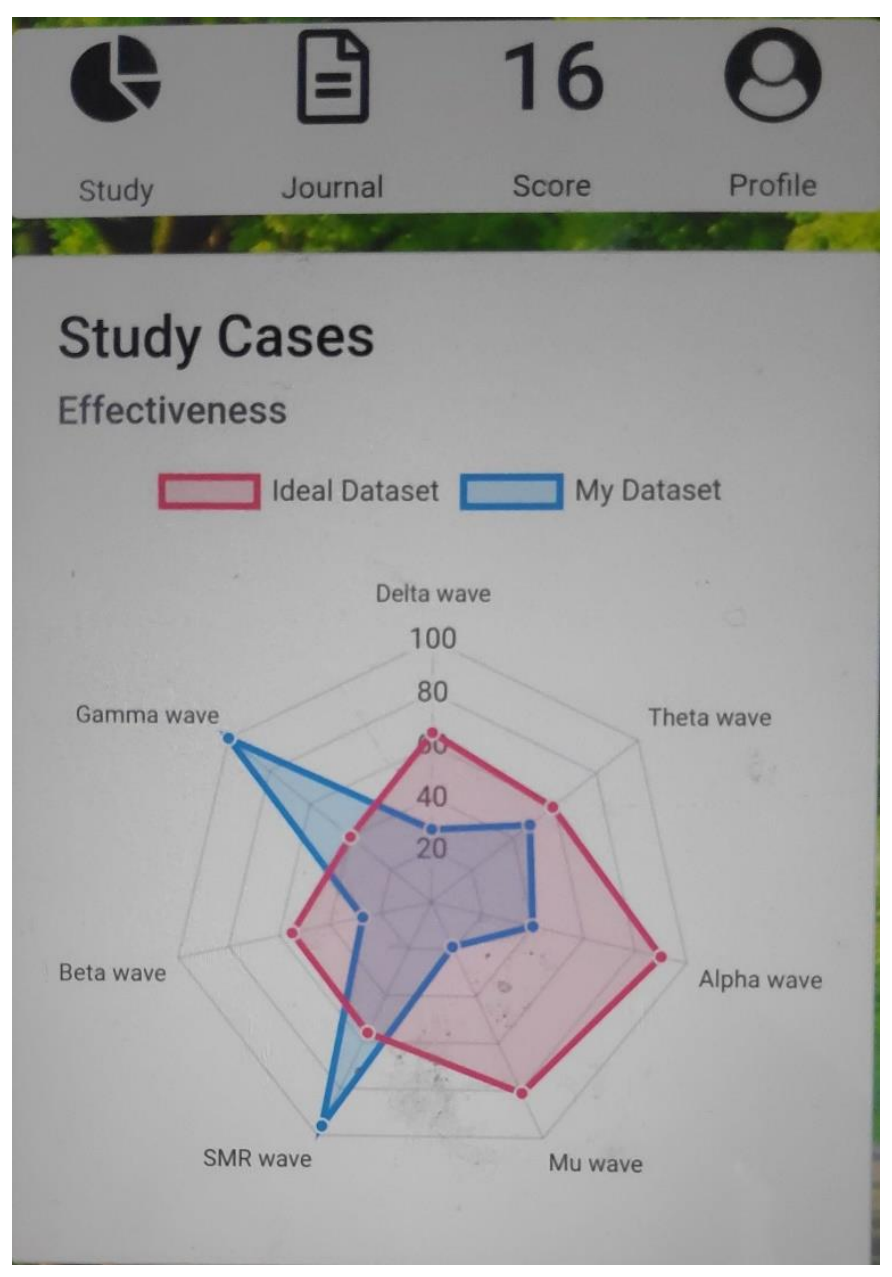

Fig. 10. Android Application Screenshot

\section{LIMITATIONS}

Current Systems provide help for people who needed help in health-related problems with eye and brain disorders and help people who are active in sports background for selecting which candidate is better among all the candidates. A limitation is the battery voltage fluctuations change the results and sweating affects the results.

\section{CONCLUSION}

Brain is made up of neuron and it is most important part of our body. After Performing this experiment, a user is comfortable while performing this test. Interface mode is a Bluetooth portable device that is easily available on any type of cell phone. This test gives the Concentration and meditation level of a person, which is used in health and sports activity to increase performance. In the future, we can also do a consultation with the help of a machine learning algorithm. 


\section{REFERENCES}

[1] Kenji Y, Junichi H, Michio M. Development of EOGBased Commu- nication System Controlled by EightDirectional Eye Movements[C]. Engineering in Medicine and Biology Society, EMBS '06, 28th Annual International Conference of the IEEE, 2006: 25742577.

[2]S. Venkataramanan, Pranay Prabhat, Shubhodeep Roy Choudhury, Har- shal B. Nemade, J.S. Sahambi, Biomedical Instrumentation based on EOG Signal Processing and Application to a Hospital Alarm System[C], proc.of IEEE ICISIP 2005:535 540, Chennai, India..

[3]Yoshitsugu Yasui, A Brainwave Signal Measurement and Data Process- ing Technique for Daily Life Applications[J]. Physiological Anthropol- ogy, 2009: 145150.

[4]Genaro Rebolledo-Mendez, Ian Dunwell and Sara de Freitas, Assess- ing NuroSky's usability to detect attention levels in an assessment exercise[C]. Human-Computer Interaction, Part1, HCII 2009, LNCS 5610:149 158.

[5]Debener S, Minow F, Emkes R, Gandras K, de Vos M. How about taking a low-cost, small, and wireless EEG for a walk? Psychophysiology. 2012. 49:1617-1621.

[6]Adarsh Rajesh, and Megha Mantur,Eyeball Gesture Controlled Auto- matic Wheelchair Using Deep Learning.2017 IEEE Region 10 Human- itarian Technology Conference (R10-HTC) 21 - 23 Dec 2017, Dhaka,

Bangladesh

[7]Sitthichai Iampetch,Yunyong Punsawad,EEG-based Mental Fatigue Pre- diction for Driving Application.The 2012 Biomedical Engineering In- ternational Conference (BMEiCON-2012).

[8]Matthew K. Mukerjee,NeuroPhone: Brain-Mobile Phone Interface using a Wireless EEG Headset.Dartmouth College, Hanover, NH, USA.

[9]Min Lin,Bin Li,A Wireless EOG-based Human Computer Interface.2010 3rd International Conference on Biomedical Engineering and Informat- ics (BMEI 2010).

[10]https:https://www.sinhaclinic.com/what-are-brainwaves/. [11]https://www.sciencedirect.com/topics/agricultural-andbiological- sciences/brain-waves.

12]https://emedicine.medscape.com/article/1139332-

overview. 